\title{
Direct carotid puncture in acute ischaemic stroke intervention
}

\author{
Elisa Colombo @ , Lorenzo Rinaldo, Giuseppe Lanzino
}

To cite: Colombo E, Rinaldo L, Lanzino G. Direct carotid puncture in acute ischaemic stroke intervention. Stroke \& Vascular Neurology 2020;5: e000260. doi:10.1136/svn2019-000260

Received 15 July 2019 Revised 9 November 2019

Accepted 8 January 2020 Published Online First 29 January 2020

\section{Check for updates}

(C) Author(s) (or their employer(s)) 2020. Re-use permitted under CC BY-NC. No commercial re-use. See rights and permissions. Published by BMJ.

Neurologic Surgery, Mayo Clinic, Rochester, Minnesota, USA

Correspondence to

Dr Giuseppe Lanzino;

lanzino.giuseppe@mayo.edu

\section{ABSTRACT}

Endovascular intervention for acute ischaemic stroke care is mostly performed in older patients, often with unfavourable aortic and supra-aortic anatomy, as well as cardiovascular comorbidities. A significant subset of them may benefit from transcervical access as the initial approach for mechanical thrombectomy. In fact, direct carotid artery puncture in these cases has the advantage to bypass the anatomical obstacles and achieve faster reperfusion. Caution is advised when common carotid artery access is pursued in order to avoid adverse events, including haematoma formation, iatrogenic arterial dissection and sheath kinking. In spite of potential complications, direct carotid puncture in acute ischaemic stroke intervention overcomes challenging angioarchitecture and may reduce the rate of poor clinical outcomes associated with delayed revascularisation in certain cases.

\section{INTRODUCTION AND HISTORICAL PERSPECTIVES}

Direct carotid puncture does not represent a new technique. In the early 1960s, when the initial cerebral angiography procedures were performed, direct puncture of the common carotid, ${ }^{1}$ the vertebral or the brachial artery was the typical access method. Cerebral angiography was predominantly performed through direct puncture of the common carotid artery (CCA) under direct vision. ${ }^{2}$ Evolution of the technique led to percutaneous puncture.

In the late 1960s and early 1970s, transfemoral catheter placement quickly replaced direct sticks of the common carotid and brachial arteries. This made it possible to study selectively the aortic branches going to the brain. Furthermore, transfemoral access proved to carry fewer and less dangerous complications. For instance, as much as groin haematomas may be painful and unpleasant for patients, they are innocuous unless they involve the retroperitoneal space. This is not true for neck haematomas, which may increase the risk for cranial neuropathies, but are mostly worrisome for rapid airway compromise requiring urgent intubation or tracheostomy. Currently, femoral artery access is the most common access route used for endovascular procedures. ${ }^{34}$
Nevertheless, aortic arch and supra-aortic vessels' anatomy presents variations and severe tortuosity in many patients, hampering endovascular treatment through transfemoral access. The most common anatomical variant of the aortic arch is the so-called bovine arch, which occurs when the left CCA shares its origin or arises directly from the right brachiocephalic artery. The prevalence of this aortic arch variant in the general population is estimated to range between $10 \%$ and $20 \% .^{5}$ When the aortic arch is defined as type II or type III, it means that the distance between the origin of the brachiocephalic trunk and the top of the aortic arch is increased. Transfemoral catheter access to the aortic arch may also be impossible in the setting of severe peripheral arterial disease. It has been reported that difficult anatomy of the aforementioned vessels causes failure in 4\%-6\% of endovascular procedures. ${ }^{6-8}$ To overcome these difficulties, direct cervical puncture of the carotid artery has been reconsidered by several authors in the setting of elective procedures. $^{6-11}$

Endovascular thrombectomy by means of manual aspiration or stent retrievers represents the favoured therapeutic strategy for acute ischaemic stroke secondary to large vessel occlusion. Typical patients who present with intracranial vessel occlusive disease are old, and in a significant subset of them arterial tortuosity makes intracranial access challenging. Prolonged and repeated attempts to catheterise a supra-aortic vessel in these patients by femoral approach may be dangerous and carries an important risk of vessel dissection and potential embolic complications. ${ }^{6}$ Moreover, an estimate of $5 \%$ of patients with difficult access to the carotids may suffer worse neurological outcomes. ${ }^{12}$

Although the transfemoral approach remains the mainstay for endovascular thrombectomy, given its success rate and safety, in a few patients, intracranial vessel catheterisation is hindered by aortic arch anatomy, carotid artery tortuosity or peripheral vessel disease. In these specific cases, consideration of direct common carotid approach is 


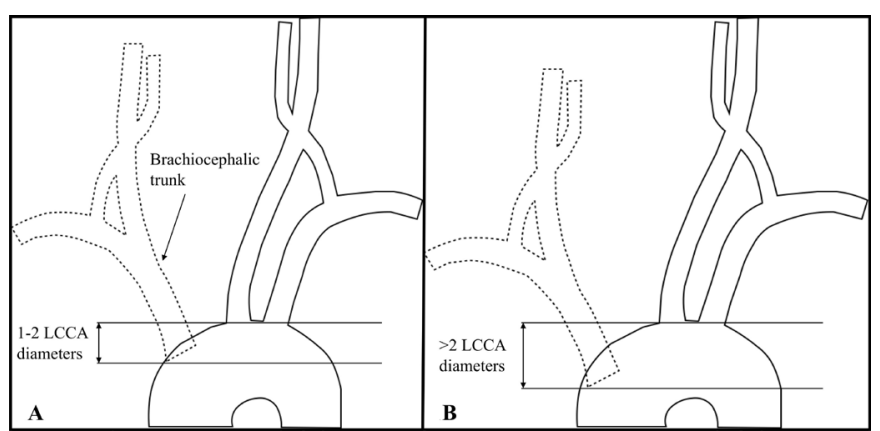

Figure 1 Graphic representation of a type II aortic $\operatorname{arch}(\mathrm{A})$ and a type III aortic arch (B). These anatomical variations of the arch are defined by the vertical distance of the brachiocephalic trunk origin and the top of the arch. Specifically, in a type II aortic arch, the distance is 1-2 diameters of the left common carotid artery (LCCA), whereas in a type III arch the distance is $>2$ LCCA diameters.

worth exploring to reduce the ominous outcomes related to difficult thrombus access through the transfemoral approach.

\section{TRANSCAROTID ARTERY REVASCULARISATION}

Recently, the hybrid procedure of transcarotid artery revascularisation (TCAR) has emerged as a valid alternative to both open carotid endarterectomy and transfemoral carotid revascularisation.

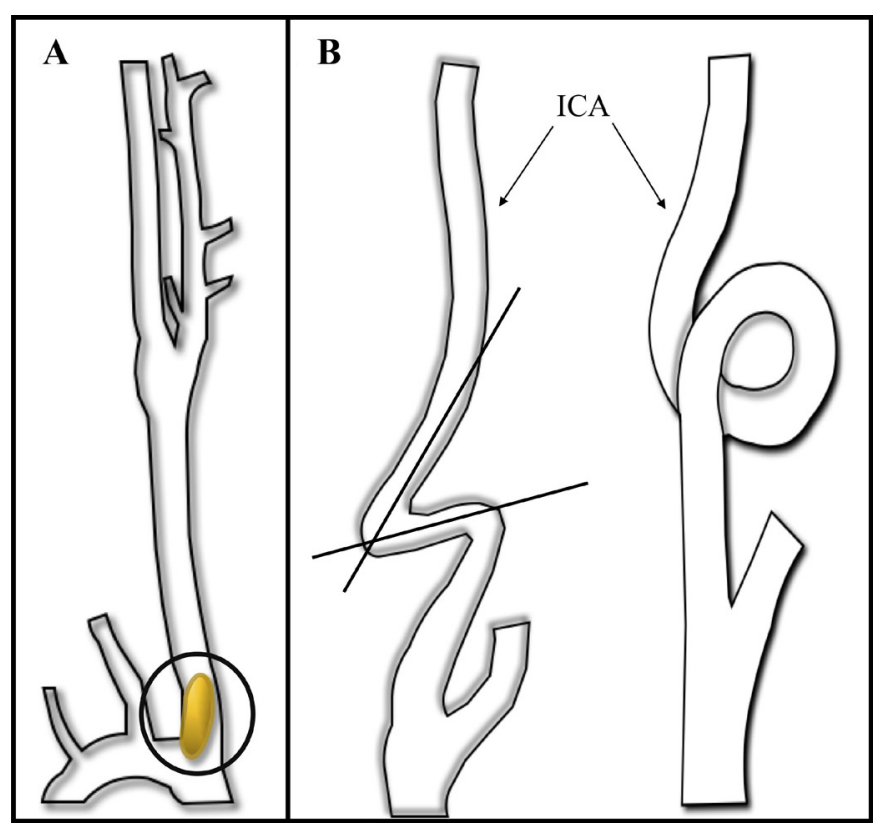

Figure 2 Carotid ostium stenosis $(A)$ is usually induced by progressive atherosclerosis and/or the intramural build-up of a plaque (black circle). Kinking and coiling of the internal carotid artery (ICA) ( $\mathrm{B}$, left and right images, respectively) are among the diverse anomalies of this vessel. Kinking is seen most frequently among elderly people, men in particular, whereas coiling is more frequent among women. When these forms are acquired, they are associated with ageing, hypertension and the usual cardiovascular risk factors.
Several studies have validated this technique, mainly for patients with difficult vascular anatomy of the aortic arch and the supra-aortic vessels. ${ }^{13-15}$ Difficult anatomical variations included type II and III aortic arches (figure 1), CCA ostium stenosis, severe internal carotid artery (ICA) tortuosity (figure 2), and also severely calcified aorta, the presence of a mural thrombus or a non-calcified plaque in the arch. Patients who may benefit from this approach are also those who suffer from comorbidities that make surgery and the transfemoral access to the carotid difficult or impossible. ${ }^{16}$ The applicability of TCAR in the general population is still not defined, but according to major publications on the topic patients are chosen on the basis of anatomical considerations. ${ }^{13} 14$

Although transfemoral carotid revascularisation offers a less invasive alternative to access the supra-aortic vessels and lowers the morbidity related to surgery, TCAR is preferred over the transfemoral approach because the latter carries a higher burden of possible embolic complications and thus periprocedural strokes in the setting of elective carotid revascularisation. ${ }^{13}{ }^{16-19}$ Furthermore, a significant subset of patients suffering from large vessel occlusive disease present vessels which are challenging to approach through the transfemoral route.

Eligibility to TCAR is mostly based on the diameter of the CCA, which must be above $6 \mathrm{~mm}$, an acceptable distance of more than $5 \mathrm{~cm}$ between the clavicle and the bifurcation, an ICA with a diameter between 4 and $9 \mathrm{~mm}$, and a depth of CCA over $4 \mathrm{~mm} .{ }^{1416}$ These parameters are relevant for technical reasons, given, for instance, the difficulty in obtaining CCA access in deep arteries. The depth of the CCA must also be considered in light of the increased risk of dissections and access site complications.

Patients shown to benefit the most from this approach are of older age. This observation may be explained by the paraphysiological changes of the ageing process on the vessels. In fact, senile carotid arteries have a tendency to develop aberrancies that may preclude a safe catheterisation through the femoral artery.

The introduction of TCAR procedure extending its application to acute ischaemic stroke care has the potential to solve many concerns regarding patients with a challenging anatomy and unfavourable long-term clinical outcomes due to long revascularisation times.

\section{PATIENT SELECTION}

In the acute setting of large vessel ischaemic stroke, prompt diagnosis and appropriate treatment are fundamental. Several studies endorsed the time urgency of stroke care, administration of intravenous tissue plasminogen activator and the fundamental role of procedure time as major determinants of long-term clinical outcomes.

Patients who present difficult supra-aortic vessel access due to anatomical or pathological variants are known to have significantly longer endovascular procedure times, which are associated with poorer neurological outcomes. ${ }^{12}$ 
The vast majority of patients treated for ischaemic stroke are advanced in age and often present a tortuous angioarchitecture or peripheral vessel disease. Therefore, they represent the category of subjects who benefit the most from direct carotid access due to difficult or even impossible thrombus access through transfemoral approach.

Direct percutaneous carotid puncture can bypass the unfavourable anatomy and reduce the time from puncture to revascularisation. The choice to pursue transcervical approach in acute settings is based on the patient's medical history, but most importantly on the appearance of the aortic arch and the supra-aortic vessels on preprocedural CT angiography (CTA). The major predictors of the ease of vessel catheterisation include arterial hypertension, hyperlipidaemia, age above 75 years and occluded vessel in the left anterior circulation. These parameters have been organised in a score by Ribo et al. ${ }^{12}$ In general, anatomical characteristics that must be considered before attempting the transfemoral approach are bovine arch, a type II or III aortic arch, and carotid dolichoarteriopathy.

Several authors validated the transcervical direct carotid approach for elective neuroendovascular procedures like carotid stenting and aneurysm coiling, but very few cases of thrombectomy through carotid puncture are reported in the literature.

In 2014 a retrospective analysis of a small case series was published by Jadhav and colleagues. ${ }^{20}$ They identified seven patients who underwent acute endovascular reperfusion via the transcervical approach. Transfemoral access was initially attempted in all patients but one, in which extreme vessel tortuosity was observed on CTA. In one patient, the transfemoral approach was abandoned due to a bovine aortic arch, whereas in the remaining five cases the authors switched to a transcarotid approach due to impossible common carotid catheterisation with multiple wires. The quality of recanalisation was good for most patients, with high rates of survival and low rates of complications related to the procedure. In this retrospective study, percutaneous puncture and catheterisation of the CCA proved instrumental in achieving quick and successful revascularisation of the intracranial vessels. The authors argued that an earlier identification of the patients as poor candidates for transfemoral approach would have prompted a transcarotid access as initial strategy. Among their conclusions, they suggested that future studies should focus on the more effective triage of the subset of patients who could benefit from a transcervical approach in acute stroke care.

The following year, Mokin and colleagues ${ }^{21}$ reported two cases in which the preprocedure CTA demonstrated a type III aortic arch and severe tortuosity of the proximal CCA ipsilateral to the occlusion. Therefore, based on the anatomical findings, the authors chose the transcervical approach as the initial access strategy. They concluded that for patients with difficult aortic arch and supra-aortic vessel anatomy, direct carotid access is 'rapid and straightforward, greatly decreasing recanalization times and increasing effectiveness'. They also emphasised the utility of preprocedure CTA for preliminary anatomy evaluation.

Roche et al gave two important contributions in $2017^{22}$ and $2019,{ }^{23}$ publishing a total of 12 cases, 11 of which reported successful direct carotid access. In 2017 they reported a case of a 73-year-old woman known to have severe stenosis of the aortic arch proximal to the subclavian artery accompanied with a poststenotic aneurysm of $8 \mathrm{~cm}$. Furthermore, she had a $2.8 \mathrm{~cm}$ aneurysm of the distal right brachiocephalic trunk and her left ICA was chronically occluded. Therefore, due to the complexity of the anatomy and the aortic coarctation, a direct percutaneous carotid puncture of the right distal CCA was chosen as access for thrombectomy. With concomitant tissue plasminogen activator (tPA), recanalisation was evaluated as Thrombolysis in cerebral infarction (TICI) 3 and the patient made a full neurological recovery. Two years later, Roche and colleagues ${ }^{22}$ published a case series of 11 patients with anterior circulation stroke managed with direct carotid puncture. Transcervical approach was not performed as initial strategy but following failure of femoral access due to unfavourable anatomy of the aortic arch, tortuosity of the supra-aortic vessels and severe atherosclerosis. Haemostasis was achieved in all patients but one by means of a closure device. Complications related to the access included one patient who demonstrated iatrogenic dissection of the vessel and developed a neck haematoma after the procedure. Overall, successful carotid access and recanalisation was achieved for most patients. The experience of Roche and colleagues proved that direct carotid access can be used in emergency settings. It also highlighted the usefulness of preprocedural CT angiogram to screen for patients with difficult anatomy and predict who could benefit from a transcervical approach rather than the standard femoral access.

Castaño $e t a l^{24}$ reported a case of successful revascularisation of left middle cerebral artery (MCA) occlusion in an 80-year-old female patient. After multiple attempts to stably catheterise the CCA ipsilateral to the thrombus, the authors changed to a transcarotid approach and were quickly able to obtain a TICI 3 recanalisation and no complications related to the procedure. The patient was dismissed from the hospital a few days after the procedure with no neurological or functional deficits. This report further emphasised the difficulty in achieving stable access to the supra-aortic vessels through the femoral approach in a subset of patients with challenging vascular anatomy. For these patients, direct carotid access is feasible and efficacious to achieve fast revascularisation and good long-term clinical outcomes. The authors endorsed again the need for future studies focusing on better screening of patients most likely to benefit from a transcervical approach.

An interesting recent case report by Benichi and colleagues $^{25}$ documented successful recanalisation of a left MCA occlusion in a 60-year-old man whose ipsilateral CCA was completely closed and the ICA was opacified 
through a Bosniak node anastomosis. A Bosniak node represents a peculiar situation in which an anastomosis is established between the external carotid artery(ECA), the cervical soft tissue vascularisation, and the arteries coming from the vertebral and ascending and deep cervical vessels. Direct access of the left carotid artery was the only option to perform mechanical thrombectomy, and puncture was performed proximal to the carotid bulb for the complex angioarchitecture. At the end of the procedure, they obtained a TICI 3 revascularisation, and a limited dissection of the proximal ICA occurred but was managed conservatively with no sequelae. Benichi and colleagues ${ }^{25}$ endorsed the validity of direct carotid access in the setting of acute stroke care in patients presenting challenging supra-aortic vessel anatomy at preprocedure imaging.

A recent case of direct surgical exposure was reported by Lin and colleagues. ${ }^{26}$

It is important to emphasise that all patients mentioned in the above studies were in their sixth, seventh and eighth decades of life. They all presented a difficult anatomy of the aortic arch, remarkable tortuosity of the supra-aortic vessels or severe atherosclerotic disease.

Furthermore, all authors agreed that a better early triage of these patients is one of the goals that must be achieved in the future in order to reduce the time from symptomatic onset and revascularisation, and consequently improve long-term functional outcomes.

A review of the literature searching for cases of direct carotid puncture in acute ischaemic stroke settings results in 24 cases published by different authors. ${ }^{21-26}$ Patient demographics and data relative to the stroke event are summarised in table 1.

\section{TECHNIQUES}

In the early times of angiography procedures, access to the carotid artery was achieved by means of puncture under direct visualisation. ${ }^{27}$ The needles used were similar to those used for femoral punctures and were exploited as the vehicle to inject for angiography, without an intermediate catheter. In case of dissection, the needle was removed, manual pressure was applied and another puncture was attempted inferiorly to the previous one. In the old days, closure of the puncture site was performed only manually.

Technological advances led to the introduction of ultrasound (US) guidance to target the site of puncture of the CCA, avoid the ansa cervicalis and verify the patency of the distal carotid on manual compression. Nowadays, a vast array of needles are available, and the procedure is made safer and less traumatic thanks to the use of dilators and guidewires. Furthermore, the introduction of sheaths increased catheter stability during the procedure.

Direct percutaneous carotid puncture can be performed under conscious sedation or general anaesthesia. The head of the patient is turned to the contralateral side by $10^{\circ}-15^{\circ}$ to raise the carotid to a more superficial position.
A pillow or a rolled towel is placed underneath the shoulders to increase neck extension. The anterior neck is then prepped in a sterile fashion and local anaesthetic is injected. The puncture site on the CCA can be assessed by palpation between the index and the middle finger or with the aid of US. Ideally, CCA puncture should be $2-3 \mathrm{~cm}$ above the superior edge of the clavicle, which is approximately at the C5-C6 level (figure 3). This location allows the introduction of the needle with an angle favourable to avoid obstruction by the clavicle. ${ }^{19}$ Using a single-wall technique, the skin is punctured, and a needle is inserted at an angle of $45^{\circ}-60^{\circ}$. On detection of mild resistance and blood pulsation waves transmitted through the needle, this is advanced through the arterial wall until pronounced backflow is observed. This part of the procedure can also be performed under roadmap control using a $4 \mathrm{~F}$ access sheath. ${ }^{6}$ The needle is then removed, and a soft guidewire is introduced into the ICA as far as possible, for most patients within the petrous segment of the vessel, ${ }^{2}$ under fluoroscopic control. The sheath is advanced over the wire and into the artery to obtain a stable position, then secured to the skin with stitches. This manoeuvre allows safe manipulation throughout the procedure and prevents the sheath from dislocating. Saline mixed with heparin is perfused during the procedure and systemic heparinisation is administered intravenously according to the patient's weight (100 units per kilogram) and coagulation profile. This is done in order to prevent thromboembolism and reduce the risk of periprocedural thromboembolic events. In addition, prevention of air embolism with careful flushing must also be observed.

Mechanical thrombectomy can either be performed my means of a direct aspiration first pass technique or with the aid of stent retrievers.

Before removal of the sheath, the patient's activated coagulation time (ACT) must be assessed. ACTs should be lower than 130 s to facilitate safe removal, sometimes requiring the use of protamine sulfate to reverse the effect of heparin if necessary. After the sheath is removed, closure of the puncture site and haemostasis can be achieved by manual compression for $15-30 \mathrm{~min}$ or the use of a closure device.

Direct access to the carotid artery can also be pursued through a surgical cut-down technique. Patient positioning and neck prepping are the same used for percutaneous puncture of the carotid artery. For this procedure, a single dose of antibiotic must be administered intravenously before starting. Three main steps characterise surgical CCA exposure. A longitudinal incision is performed along the anterior border of the sternocleidomastoid muscle for a length of 3-4cm. Then, the platysma is identified and dissected. The third major step in the exposure is the identification of the medial border of the internal jugular vein. The carotid sheath is medial to the jugular vein hallmark and is exposed after dividing veins running medially. The CCA is then isolated proximally and distally to the chosen puncture site and a vessel 


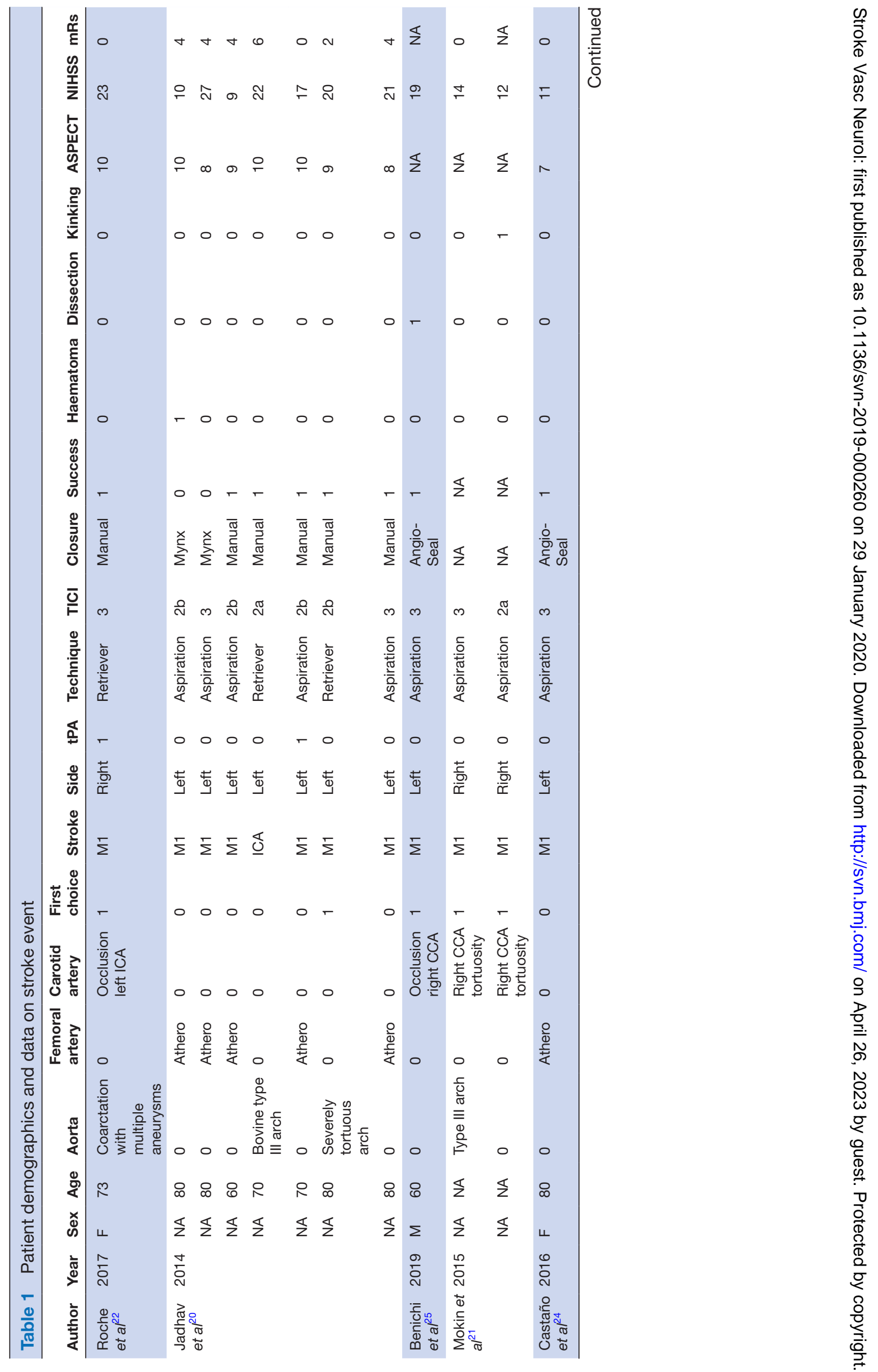




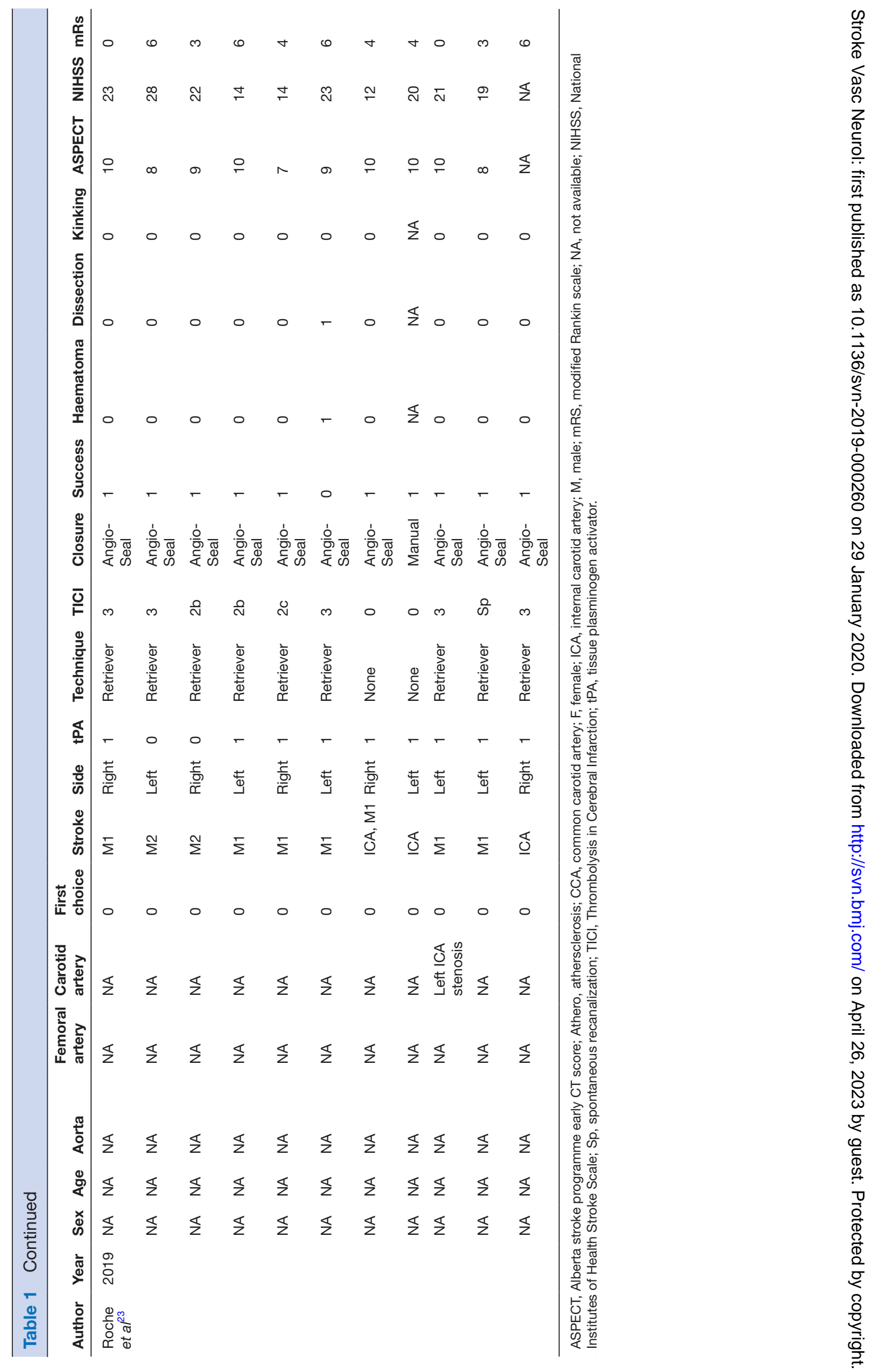


NOFGUREDOUNDFigure 3 Direct percutaneous carotid puncture can be performed under conscious sedation or general anaesthesia with the head turned to the contralateral side by $10^{\circ}-15^{\circ}$. The puncture site on the CCA can be assessed by palpation between the index and the middle finger or with the aid of ultrasound. Ideally, CCA puncture should be $2-3 \mathrm{~cm}$ above the superior edge of the clavicle, which is approximately at the C5-C6 level (black star). The skin is punctured, and a needle is inserted at a $45^{\circ}-60^{\circ}$ angle. This part of the procedure can also be performed under roadmap control using a $4 \mathrm{~F}$ access sheath. CCA, common carotid artery.

loop is placed around it. The needle is then advanced with a flat angle into the vessel under visual control and a purse-string suture is placed at the puncture site. The remaining steps recapitulate those of the percutaneous puncture of the carotid artery (figure 4). At the end of the procedure, on removal of the sheath, haemostasis is achieved by tightening the purse-string suture. After haemostasis is obtained, the skin is closed in layers and the patency of the artery is confirmed by Doppler US.

Manual compression of the access site is the most used method to achieve haemostasis after an angiographic procedure. $^{2610}$ Percutaneous closure devices are a relatively new introduction in the field. Nevertheless, several reviews demonstrated their success and safety for femoral artery access closure, ${ }^{28}$ even in patients on antiplatelet and anticoagulation therapy. ${ }^{29}$

The most studied transfemoral closure device, used also for the cervical arteries, is the Angio-Seal (St Jude Medical, St Paul, Minneapolis, USA). This device comes in $6 \mathrm{~F}$ and $8 \mathrm{~F}$ sizes and comprises a collagen sponge and a specifically designed polymer anchor $(50 \%$ lactide and $50 \%$ glycolide copolymer) connected by a self-tightening

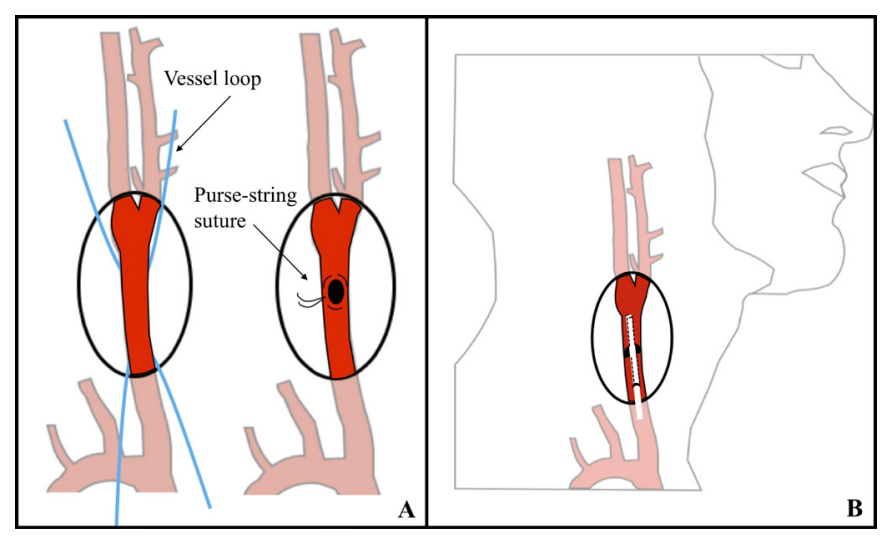

Figure 4 When surgical cut-down technique is chosen to gain direct access to the carotid artery, the common carotid artery is isolated proximally and distally to the chosen puncture site and the vessel loops are placed around it (A). The needle is then advanced with a flat angle into the vessel under visual control and a purse-string suture is placed at the puncture site (A). The remaining steps recapitulate those of the percutaneous puncture of the carotid artery (B). suture. All three of these components are absorbable. This device occupies $4 \%$ of the lumen of the artery; the anchor softens by water absorption immediately after the placement and is followed by encapsulation by endothelial cells within 7 days and absorption with healing in less than 90 days from the procedure. The collagen plug, which forms in contact with the adventitia, is resorbed after a longer period of time. ${ }^{10}$

Another transfemoral tool, used for a transcervical procedure, is the StarClose Extravascular Closure System (Abbott, Santa Clara, USA). It is constituted by a nitinol clip, which is designed to promote primary healing of the arterial wall.

Their application for carotid artery access closure is still debated, few cases have been published in the literature, and no device has gained formal approval to cervical carotid closure. Moret's group has reported percutaneous access to the carotid artery followed by closure of the artery using an Angio-Seal device in a handful of patients. ${ }^{910}$ Successful closure of a carotid artery puncture with the same device has also been reported by Massiere and colleagues ${ }^{30}$ and Nii and colleagues. ${ }^{28}$ Furthermore, in the setting of acute ischaemic stroke intervention by means of direct carotid access, the use of the Angio-Seal has been reported by Mokin et al. ${ }^{21}$ The use of a Mynx device (Cordis, Cardinal Health, Dublin, Ireland) has been published by Jadhav and colleagues ${ }^{20}$ but proved unsuccessful and resulted in haematoma formation. This device closes the vessel by synergistic action of a temporary balloon, inflated into the lumen of the vessel, and a special sealant which effectively grips the arterial wall and reabsorbs in 30 days.

In general, deployment of a percutaneous closure device carries the risk of haematoma formation and ischaemic events due to potential migration of the intraluminal anchor. Therefore, the use of these devices for carotid artery closure must be carefully managed by a well-trained neurointerventionalist.

\section{COMPLICATIONS}

In general, there are three main complications related to percutaneous puncture of an arterial vessel: failed haemostasis after sheath removal and thus haematoma formation, iatrogenic dissection of the vessel, and intraluminal kinking of the sheath. Clinical significance of these adverse events is determined by their extent and most importantly by the anatomical region of the vessel. For instance, groin haematomas can be very unpleasant, but do not represent a life-threatening complication, unless there is involvement of the retroperitoneal space. On the contrary, neck haematomas may cause rapid and severe airway compromise, often requiring urgent intubation, and may be fatal.

Transfemoral access gained approval as the mainstay for angiography procedure given its lower rate of complication in comparison with the classic transcervical and 
transbrachial access routes. ${ }^{78}$ Nonetheless, direct carotid puncture carries a relatively low risk of complications.

The most relevant and common adverse event associated with percutaneous carotid puncture is the formation of haematomas. Previous series have documented a $4 \%-7 \%$ rate of neck haematoma after sheath removal in percutaneous carotid puncture. ${ }^{9}$ Neck haematoma may cause important mass effect on the trachea and compromise airway patency. Moreover, haematoma formation in this region increases the risk of cranial neuropathies. Open carotid access using a cut-down approach achieves better haemostasis and decreases the chance of haematoma formation. ${ }^{10}{ }^{31}$ In addition, the advent of percutaneous closure devices allows better haemostasis and reduces postprocedural morbidity since patients are ambulatory earlier than after closure with manual compression. Furthermore, closure devices reduce the risk of adverse events also for patients on heavy regimens of anticoagulant and antiplatelet agents. ${ }^{32}$

Sheath kinking occurs when puncture of the CCA lies too close to the clavicle and therefore the entry direction of the sheath is almost perpendicular to the course of the artery. Kinking of the sheath may damage the vessel. In case mechanical thrombectomy is performed by manual aspiration, a kinked sheath also compromises the effectiveness of aspiration catheter delivery and manoeuvrability.

In cases of thrombectomy by direct carotid artery puncture found in the literature (table 1), two patients $(8.7 \%)$ had a haematoma formed after the removal of the sheath. ${ }^{20}{ }^{23}$ In one of these cases, the haematoma caused some lateral displacement of the airway due to mass effect. A CTA did not show contrast extravasation from the haematoma, and neither airway support nor further interventions were required. The scenario was different for the other patient, who was electively intubated to protect the airway after observation of contrast extravasation on angiography. On a follow-up CTA, no pseudoaneurysm was seen, the patient was extubated and the haematoma was managed conservatively. In both cases, haematoma formation followed unsuccessful deployment of a percutaneous closure device.

In the reviewed cases, two patients $(8.7 \%)$ suffered iatrogenic dissection of the ICA. ${ }^{23}$ Both patients demonstrated a stable dissection and were managed conservatively. No periprocedural embolic complication was observed.

Sheath kinking was only observed in one case $(4.3 \%))^{21}$ This adverse event did not cause any damage to the carotid artery, but it did compromise the efficacy of the aspiration thrombectomy and the extent of revascularisation according to the TICI score.

Despite the associated complications, the reported cases demonstrate good quality of recanalisation with good clinical outcomes. The observed survival rates in this cohort are high and exceed those expected for this age group. Furthermore, these cases emphasise the validity of percutaneous closure devices for successful puncture site closure.

\section{CONCLUSIONS}

Transfemoral access remains the standard approach for endovascular angiography procedures. This entry route carries a lower rate of adverse events and is suitable for most patients, especially in elective settings. Nevertheless, difficult aortic arch anatomy, tortuosity of the carotid arteries or an ostial lesion, and severe peripheral vascular disease may compromise the catheterisation of the supraaortic vessels and even make it impossible.

Endovascular intervention for acute ischaemic stroke care is mostly performed in older patients, often with unfavourable aortic and supra-aortic anatomy, as well as cardiovascular comorbidities. A significant subset of them may benefit from transcervical access as the initial approach for mechanical thrombectomy. In fact, direct carotid artery puncture in these cases has the advantage to bypass the anatomical obstacles and achieve faster reperfusion. Caution is advised when CCA access is pursued in order to avoid adverse events, including haematoma formation, iatrogenic arterial dissection and sheath kinking.

In spite of potential complications, direct carotid puncture in acute ischaemic stroke intervention overcomes challenging angioarchitecture and may reduce the rate of poor clinical outcomes associated with delayed revascularisation in certain cases.

Contributors GL: interpreted the data and revised the manuscript for intellectual content. LR: revised the manuscript for intellectual content. EC: drafted the manuscript for intellectual content.

Funding The authors have not declared a specific grant for this research from any funding agency in the public, commercial or not-for-profit sectors.

Competing interests None declared.

Patient consent for publication Not required.

Provenance and peer review Commissioned; externally peer reviewed.

Open access This is an open access article distributed in accordance with the Creative Commons Attribution Non Commercial (CC BY-NC 4.0) license, which permits others to distribute, remix, adapt, build upon this work non-commercially, and license their derivative works on different terms, provided the original work is properly cited, appropriate credit is given, any changes made indicated, and the use is non-commercial. See: http://creativecommons.org/licenses/by-nc/4.0/.

ORCID iD

Elisa Colombo http://orcid.org/0000-0003-0424-1250

\section{REFERENCES}

1 Leeds NE, Kieffer SA. Evolution of diagnostic neuroradiology from 1904 to 1999. Radiology 2000;217:309-18.

2 Hassan AE, Chaudhry SA, Miley JT, et al. Microcatheter to recanalization (procedure time) predicts outcomes in endovascular treatment in patients with acute ischemic stroke: when do we stop? AJNR Am J Neuroradiol 2013;34:354-9.

3 Al-Mubarak N, Roubin GS, Vitek JJ, et al. Procedural safety and short-term outcome of ambulatory carotid stenting. Stroke 2001;32:2305-9.

4 Koenigsberg RA, Aletich V, Camras L, et al. Direct cervical internal carotid access for GDC treatment of an ophthalmic origin carotid aneurysm. Surg Neurol 1999;51:506-8.

5 Brott TG, Hobson RW, Howard G, et al. Stenting versus endarterectomy for treatment of carotid-artery stenosis. N Engl J Med 2010;363:11-23.

6 Dorfer C, Standhardt H, Gruber A, et al. Direct percutaneous puncture approach versus surgical cutdown technique for intracranial 
neuroendovascular procedures: technical aspects. World Neurosurg 2012;77:192-200.

7 Bavinzski G, Killer M, Ferraz-Leite H, et al. Endovascular therapy of idiopathic cavernous aneurysms over 11 years. AJNR Am J Neuroradiol 1998;19:559-65.

8 Bederson JB, Awad IA, Wiebers DO, et al. Recommendations for the management of patients with unruptured intracranial aneurysms: a statement for healthcare professionals from the stroke Council of the American heart association. Stroke 2000;31:2742-50.

9 Blanc R, Piotin M, Mounayer C, et al. Direct cervical arterial access for intracranial endovascular treatment. Neuroradiology 2006;48:925-9.

10 Blanc R, Mounayer C, Piotin M, et al. Hemostatic closure device after carotid puncture for stent and coil placement in an intracranial aneurysm: technical note. AJNR Am J Neuroradiol 2002;23:978-81.

11 Mathieu X, Piret V, Bergeron P, et al. Choice of access for percutaneous carotid angioplasty and stenting: a comparative study on cervical and femoral access. J Cardiovasc Surg 2009;50:677-81.

12 Ribo M, Flores A, Rubiera M, et al. Difficult catheter access to the occluded vessel during endovascular treatment of acute ischemic stroke is associated with worse clinical outcome. J Neurointerv Surg 2013:5:i70-3.

13 Lukins DE, Pilati S, Escott EJ. The moving carotid artery: a retrospective review of the retropharyngeal carotid artery and the incidence of positional changes on serial studies. AJNR Am J Neuroradiol 2016;37:336-41.

$14 \mathrm{Han} \mathrm{H}-\mathrm{C}$. Twisted blood vessels: symptoms, etiology and biomechanical mechanisms. J Vasc Res 2012;49:185-97.

15 Campeau L. Percutaneous radial artery approach for coronary angiography. Cathet Cardiovasc Diagn 1989;16:3-7.

16 Eckstein $\mathrm{H}-\mathrm{H}$, Ringleb P, Allenberg J-R, et al. Results of the StentProtected angioplasty versus carotid endarterectomy (space) study to treat symptomatic stenoses at 2 years: a multinational, prospective, randomised trial. Lancet Neurol 2008;7:893-902.

17 Mas J-L, Arquizan C, Calvet D, et al. Long-Term follow-up study of endarterectomy versus angioplasty in patients with symptomatic severe carotid stenosis trial. Stroke 2014;45:2750-6.

18 Malas MB, Leal J, Kashyap V, et al. Technical aspects of transcarotid artery revascularization using the ENROUTE transcarotid neuroprotection and stent system. J Vasc Surg 2017;65:916-20.

19 Kwolek CJ, Jaff MR, Leal JI, et al. Results of the ROADSTER multicenter trial of transcarotid stenting with dynamic flow reversal. $J$ Vasc Surg 2015;62:1227-34.
20 Jadhav AP, Ribo M, Grandhi R, et al. Transcervical access in acute ischemic stroke. J Neurointerv Surg 2014;6:652-7.

21 Mokin M, Snyder KV, Levy El, et al. Direct carotid artery puncture access for endovascular treatment of acute ischemic stroke: technical aspects, advantages, and limitations. J Neurointerv Surg 2015;7:108-13.

22 Roche AD, Murphy B, Adams N, et al. Direct common carotid artery puncture for endovascular treatment of acute large vessel ischemic stroke in a patient with aortic coarctation. J Stroke Cerebrovasc Dis 2017;26:e211-3.

23 Roche A, Griffin E, Looby S, et al. Direct carotid puncture for endovascular thrombectomy in acute ischemic stroke. J Neurolnterv Surg 2019;0:1-7.

24 Castaño C, Remollo S, García MR, et al. Mechanical thrombectomy with 'ADAPT' technique by transcervical access in acute ischemic stroke. Neuroradiol J 2015;28:617-22.

25 Benichi S, Consoli A, Coskun O, et al. Thrombectomy by direct cervical access in case of common carotid occlusion with collateral supply to internal carotid artery: uncommon anatomic variant. World Neurosurg 2019;124:84-6.

26 Lin CM, Chang $\mathrm{CH}$, Chen SW, et al. Direct neck exposure for endovascular mechanical thrombectomy in a patient with acute internal carotid occlusion concurrent type A aortic dissection. World Neurosurg 2019.

27 Malas MB, Dakour-Aridi H, Wang GJ, et al. Transcarotid artery revascularization versus Transfemoral carotid artery stenting in the Society for vascular surgery vascular quality initiative. J Vasc Surg 2019;69:92-103.

28 Nii K, Kazekawa K, Onizuka M, et al. Direct carotid puncture for the endovascular treatment of anterior circulation aneurysms. AJNR Am J Neuroradiol 2006;27:1502-4.

29 Yee KOK-M, Lazzam C, Richards JAN, et al. Same-Day discharge after coronary stenting:. A feasibility study using a hemostatic femoral puncture closure device. J Interv Cardiol 2004;17:315-20.

$30 \mathrm{Kim} \mathrm{HY,} \mathrm{Choo} \mathrm{SW,} \mathrm{Roh} \mathrm{HG,} \mathrm{et} \mathrm{al.} \mathrm{Efficacy} \mathrm{of} \mathrm{femoral} \mathrm{vascular}$ closure devices in patients treated with anticoagulant, abciximab or thrombolytics during percutaneous endovascular procedures. Korean J Radiol 2006;7:35-40.

31 Massière B, von Ristow A, Cury JM, et al. Closure of carotid artery puncture site with a percutaneous device. Ann Vasc Surg 2009;23:256.e5-7.

32 Sfyroeras GS, Moulakakis KG, Markatis F, et al. Results of carotid artery stenting with transcervical access. J Vasc Surg 2013;58:1402-7. 